\title{
Comparative analysis of the RTFL peptide family on the control of plant organogenesis
}

\author{
Pin Guo • Asami Yoshimura • Naoko Ishikawa • \\ Takahiro Yamaguchi · Youhao Guo $\cdot$ Hirokazu Tsukaya
}

Received: 5 August 2014 / Accepted: 25 December 2014 / Published online: 21 February 2015

(C) The Author(s) 2015. This article is published with open access at Springerlink.com

\begin{abstract}
Plant peptides play important roles in various aspects of plant growth and development. The RTFL/DVL family includes small peptides that are widely conserved among land plants. Overexpression of six RTFL genes in Arabidopsis was suggestive of their functions as negative regulators of cell proliferation and as positional cues along the longitudinal axis of the plant body. At this time, few reports are available on RTFL paralogs in other species and the evolutionary relationship of RTFL members among land plants remains unclear. In this study, we compared and analyzed whole amino acid sequences of 188 RTFL members from 22 species among land plants and identified 73 motifs. All RTFL members could be grouped into four clades, and each clade exhibited specific motif patterns, indicative of unique evolutionary traits in the RTFL family. In agreement with this hypothesis, we analyzed two RTFL
\end{abstract}

Electronic supplementary material The online version of this article (doi:10.1007/s10265-015-0703-1) contains supplementary material, which is available to authorized users.

P. Guo $\cdot$ Y. Guo

College of Life Science, Wuhan University, Wuhan 430072,

Hubei, China

P. Guo $\cdot$ A. Yoshimura $\cdot$ N. Ishikawa $\cdot$ H. Tsukaya $(\bowtie)$ Department of Biological Sciences, Graduate School of Science, University of Tokyo, Hongo, Tokyo 113-0033, Japan

e-mail: tsukaya@bs.s.u-tokyo.ac.jp

Present Address:

N. Ishikawa

Graduate School of Arts and Sciences, The University of Tokyo,

3-8-1 Komaba, Tokyo 153-8902, Japan

T. Yamaguchi

Acel, Inc. SIC1 1201, 5-4-21 Nishihashimoto, Midori-ku,

Sagamihara, Kanagawa, Japan members from Oryza sativa and Arabidopsis by overexpressing them in Arabidopsis, revealing similar phenotypes suggestive of a conserved function of the RTFL family between eudicots and monocots, as well as different phenotypes and unique functions.

Keywords Evolution - Motif patterns - Organogenesis . Plant peptides $\cdot$ RTFL/DVL family

\section{Introduction}

To date, numerous peptides have been identified from plant genomes based on biochemical and genetic studies (Farrokhi et al. 2008; Kastin 2013). Peptides are defined as short chains of amino acid monomers; precursors are rarely larger than 120 amino acids and are typically present at low physiological concentrations (Katsir et al. 2011). Structurally, plant peptides can be categorized into two classes; secretory peptides and non-secretory peptides, based on the presence of an $\mathrm{N}$-terminal secretory signal sequence (Matsubayashi 2014). These plant peptides play important roles in many processes including defense responses, callus growth, meristem organization, root growth, leaf-shape regulation, and nodule development (Matsubayashi 2014; Matsubayashi and Sakagami 2006). The secretory peptides act as signaling molecules for intercellular communication. CLAVATA3 (CLV3), ROOT MERISTEM GROWTH FACTOR1 (RGF1), and S-Locus cysteine-rich protein (SCR) or S-locus protein 11 (SP11) are well-characterized examples of secretory peptides (Fletcher et al. 1999; Kachroo et al. 2001; Matsuzaki et al. 2010; Schopfer et al. 1999).

Non-secretory peptides also play roles in the wounding/innate immunity response, nodule development, root growth, and leaf-shape regulation (Matsubayashi 2014; 
Matsubayashi and Sakagami 2006; Sakagami 2007). The non-secretory peptides are further divided into two types based on whether they act extracellularly or intracellularly (Matsubayashi 2014). Two well-characterized non-secretory peptides (Systemin and AtPep1) are known to act in the extracellular space; namely, Systemin (tomato systemin, TomSys), the first plant peptide discovered in wounded tomato (Solanum lycopersicum) leaves that can induce the production of proteinase inhibitors to defend against herbivore and pathogen attacks (Pearce et al. 1991); and AtPep1, the first known endogenous peptide elicitor that can be induced by wounding, methyl jasmonate, or ethylene, and activates innate immune responses such as the transcription of defensin, the production of $\mathrm{H}_{2} \mathrm{O}_{2}$, and the expression of its precursor gene (Huffaker et al. 2006). Although both TomSys and Atpep1 are non-secretory peptides in structure and remain as precursors with a constitutively low expression level in the cytoplasm, the precursor proteins can be hydrolyzed into active peptides and released into the intercellular space upon cell wounding or pathogen invasion (Huffaker et al. 2006; Li et al. 2001; Meindl et al. 1998; Narváez-Vásquez and Ryan 2004; Narváez-Vásquez et al. 2005; Pearce et al. 1991; Scheer and Ryan 2002; Yamaguchi et al. 2006; Yamaguchi and Huffaker 2011). After binding to their receptors on the plasma membrane, wounding/ defense information is transduced into intact cells to induce similar defense responses (Meindl et al. 1998; Scheer and Ryan 2002; Yamaguchi et al. 2006). Comparatively, EARLY NODULIN40 (ENOD40) and POLARIS (PLS) are considered typical non-secretory peptides that are synthesized and function intracellularly (Matsubayashi 2014). ENOD40 is an early nodulin gene that is rapidly expressed during the invasion of rhizobia in the root pericycle and nodule primordium (Crespi et al. 1994; Kouchi and Hata 1993; Yang et al. 1993). Two short peptides (ENOD40A and ENOD40B) are directly translated from ENOD40 mRNA (Röhrig et al. 2002). ENOD40 peptides strongly bind the cytosolic sucrose synthase (SuSy) enzyme (Chae et al. 2012; Röhrig et al. 2004) and are thought to activate sucrose cleavage and nodule development (Charon et al. 1999; Kumagai et al. 2006; Podkowinski et al. 2009; Takeda et al. 2005; Wan et al. 2007). PLS, a short open reading frame encoding a 36 -amino-acid peptide, is required for correct auxin-cytokinin homeostasis to modulate root growth and leaf vascular patterning (Casson et al. 2002), and also negatively regulates ethylene responses to modulate cell division and expansion via the effects on cytoskeleton and auxin signaling (Chilley et al. 2006).

ROTUNDIFOLIA4 (ROT4) was isolated through a gainof-function genetic screen that resulted in a mutant called rotundifolia4-1D (rot4-1D) (Narita et al. 2004). This mutant exhibited shorter leaves with a reduced cell number mainly along the longitudinal axis in Arabidopsis. ROT4 encodes a short peptide of 53 amino acids and negatively regulates cell proliferation in the longitudinal axis of organs, resulting in a phenotype of "small-round" rosette leaves (Narita et al. 2004; Wen et al. 2004). ROT4 is believed to be a non-mobile peptide synthesized without proteolytic processing since overexpressed ROT4-GFP and GFP-ROT4 localize on the plasma membrane and confer similar phenotypes (Ikeuchi et al. 2010; Narita et al. 2004). Overexpression of ROT4 under control of the heat shock promoter constructed using the Cre/Lox recombination system (Ikeuchi et al. 2010) suggests that ROT4 works cell-autonomously, which is indicative of its non-mobile characteristics. Wen et al. (2004) also identified a gene, DEVILI (DVL1), from activation-tagged lines that develop horned fruit tips, which was later shown to be a paralog of ROT4 in Arabidopsis. In total, 22 putative homologs of ROT4 and DVL1 were identified in the Arabidopsis genome, which were designated as the ROT-FOURLIKE/DEVIL (RTFL/DVL) family (Narita et al. 2004; Wen et al. 2004; Yamaguchi et al. 2013). RTFL/DVL peptides share a highly conserved domain of approximately 30 amino acids in the C-terminus, named the RTF domain (Narita et al. 2004). Overexpression of the RTF domain is sufficient to induce the rot4- $1 D$ phenotype (Ikeuchi et al. 2010). The remaining sequences of the RTFL/DVL family (especially in the N-terminal region) are poorly conserved and studied (Ikeuchi et al. 2010; Narita et al. 2004; Wen et al. 2004). Notably, RTFL/DVL members are highly variable in the length of their amino acid sequences (40-144 amino acids in Arabidopsis), suggestive of various roles or functions.

Our understanding of the biological function of the $R T F L / D V L$ family is based on phenotypes observed in overexpression lines. Overexpression of at least six members of the RTFL/DVL family in Arabidopsis produces short-leaf phenotypes, which were similar to the rot4$1 D$ mutant (Narita et al. 2004; Wen et al. 2004). Besides leaves, pleiotropic phenotypes in lateral organs are observed among overexpressors, such as shortened floral organs, protruding structures on the valves of fruits and at the base of pedicels, as well as trichomes (Ikeuchi et al. 2010). ROT4/DVL16 suppresses polarized cell proliferation along the longitudinal axis, which mainly accounts for its effect on the shortened leaf phenotype (Ikeuchi et al. 2010) and the other lateral above-ground organs, which are considered to be leaf-derived organs (Golz and Hudson 2002; Valdivia et al. 2012). However, the loss-of-function lines provide little information on the biological function of the RTFL/DVL family. The reported insertional mutant of RTFL4/DVL15 (Narita et al. 2004) and RNA interference constructs targeting DVL1/RTFL18 and DVL3/RTFL21 (Wen et al. 2004) did not produce any noticeable loss-offunction phenotype. Narita et al. (2004) also identified two insertional mutants within Oryza homologs, which were named osrtfl1-1 and osrtfl2-1. Unfortunately, the mutants 
or mutant alleles did not show any discernable phenotypes, suggestive of a high level of genetic redundancy among the RTFL/DVL family.

The RTFL/DVL family is widely conserved among land plants (Floyd and Bowman 2007). However, the majority of studies have focused on RTFL/DVL members in Arabidopsis. The only report on a RTFL/DVL member in Medicago truncatula was published by Combier et al. (2008), which functioned as a negative factor to reduce nodulation. To explore the evolutionary processes and biological functions of this family, we compared the whole putative amino acids sequences of 188 RTFL/DVL members from liverworts to angiosperms and overexpressed one RTFL/DVL member from Oryza sativa in Arabidopsis. Comparative analysis was suggestive of an evolutionary trait of the RTFL/DVL family and revealed specific amino acids patterns (motif patterns) among species. The comparison could be used as background information for further study on the biological function of RTFL/DVL members in other species. Moreover, overexpression studies were suggestive of a conserved function of the RTFL/DVL family between monocots and eudicots in the control of plant organogenesis.

\section{Materials and methods}

Plant materials and growth conditions

The Arabidopsis accession Columbia (Col-0) was used as the wild type, and p35S::ROT4 reported in Narita et al. (2004) was used as a reference line in this report. Oryza sativa L. cv. Taichung 65 (T65), a kind gift from Prof. Hiroyuki Hirano (The University of Tokyo), was used to isolate OsRTFL3 (Os01t0972300). Transgenic lines of p35S::OsRTFL3 were generated as described below. One single T-DNA insertion line (homozygous) was used for genetic and cytological analysis. Three independent heterozygous lines of OSRTFL3 o/x were used to confirm the root defects, as shown in Supplementary Fig. 2.

All Arabidopsis plants were grown on rock wool or Murashige and Skoog (MS) medium (Gamborg et al. 1976) at $22{ }^{\circ} \mathrm{C}$ under continuous light. T65 plants were grown in the container of clay loam soil under the same conditions. Young seedlings of Arabidopsis and T65 were collected 10 days after germination for reverse transcription polymerase chain reaction (RT-PCR) analysis. Arabidopsis used for root growth studies were cultured and grown on MS medium in the vertical direction.

Vector construction and transformation

Total RNA was isolated from 10-day-old seedlings of T65 using the RNeasy Mini Kit (Qiagen, Valencia, CA,
USA). The SuperScript one-step RT-PCR kit (Invitrogen, Carlsbad, CA, USA) was used for RT-PCR according to the manufacturer's protocol. The amplification conditions of OsRTFL3 using RT-PCR was one cycle at $95{ }^{\circ} \mathrm{C}$ for $2 \mathrm{~min}$, followed by 35 cycles at $94{ }^{\circ} \mathrm{C}$ for $30 \mathrm{~s}, 55^{\circ} \mathrm{C}$ for $30 \mathrm{~s}$, and $72{ }^{\circ} \mathrm{C}$ for $1 \mathrm{~min}(2720$ Thermal Cycler; Applied Biosystems, Foster City, CA, USA). The following pair of primers was used for amplification: OsRTFL3-Fw: 5'-CACCATGGAGGACGAGAGGTGGAAGC- $3^{\prime}$ and Os RTFL3-Rev: 5'-CTAGTAGTCTCGCCAGCAGACGAG'. The RT-PCR product was cloned into the pENTRD-TOPO vector (Invitrogen) and then introduced into $\mathrm{PH} 35 \mathrm{G}$, a binary vector containing a Gateway cassette (Invitrogen) in the sense orientation under a CaMV $35 \mathrm{~S}$ promoter (Narita et al. 2004).

The construct was introduced into wild-type Arabidopsis using Agrobacterium-mediated transformation with the simplified floral dip method (Clough and Bent 1998). Transgenic plants were selected on MS medium containing $2 \mathrm{mg} \mathrm{mL}^{-1}$ Gellan Gum (Wako, Osaka, Japan) and $20 \mu \mathrm{g} \mathrm{mL}^{-1}$ Hygromycin B (Aventis Pharma Ltd., Tokyo, Japan).

\section{Genomic PCR}

Seven-day-old plants of wild type, OsRTFL3 o/x, and OsRTFL3 o/x-1-4 were used for genomic DNA isolation using the DNeasy Plant Mini Kit (Qiagen, Valencia, CA, USA). For the amplification of OsRTFL3, the following primers were used: OsRTFL3-F, 5'-ACTCGTCC GATTTCAACAGC- $3^{\prime}$ and OsRTFL3-R, 5'-GGCGGACG ATGTAGAACCT- $3^{\prime}$. The amplification conditions using genomic DNA as template was one cycle at $95{ }^{\circ} \mathrm{C}$ for 2 min, followed by 20 cycles of touchdown PCR $\left[94{ }^{\circ} \mathrm{C}\right.$ for $30 \mathrm{~s}, 57^{\circ} \mathrm{C}$ for $30 \mathrm{~s}$ (the temperature was reduced by $0.4{ }^{\circ} \mathrm{C}$ per cycle), and $72{ }^{\circ} \mathrm{C}$ for $90 \mathrm{~s}$ ] and then 20 cycles of nontouchdown PCR $\left(94{ }^{\circ} \mathrm{C}\right.$ for $30 \mathrm{~s}, 55{ }^{\circ} \mathrm{C}$ for $30 \mathrm{~s}$, and $72{ }^{\circ} \mathrm{C}$ for $90 \mathrm{~s}$ ), with a final $72{ }^{\circ} \mathrm{C}$ for $7 \mathrm{~min}$.

\section{Semiquantitative RT-PCR}

For the amplification of ROT4 and OSRTFL3, the following primers were used: OsRTFL3-F, 5'-GATTTCAACAGCAG CAACGC-3'; OsRTFL3-R, 5'-CGAATTGTTGCTCTGCT GCT-3'; ROT4-F: 5'-AGGAGAATGGCACGTGTGAG-3'; and ROT4-R: 5'-CAAGAGTCTTTGCGGTCGTG-3'. ACTIN2 (ACT2) was used as a control to detect constitutive expression. Primers for amplification of $A C T 2$ were as follows: ACT2-F, 5'-GAAATCACAGCACTTGCACC-3' and ACT2-R, 5'-AAGCCTTTGATCTTGAGAGC- $3^{\prime}$. The amplification conditions by RT-CPR was one cycle at $95^{\circ} \mathrm{C}$ for $2 \mathrm{~min}$, followed by 35 cycles at $94{ }^{\circ} \mathrm{C}$ for $30 \mathrm{~s}, 57^{\circ} \mathrm{C}$ for $30 \mathrm{~s}$, and $72{ }^{\circ} \mathrm{C}$ for $1 \mathrm{~min}$. 
Comparative and phylogenetic analysis

The database used for searching sequences of RTFL members are listed in Table 1. To select RTFL members, HMMER was used to search the Pfam database and BLASTP was used to search SALAD, Pytozome v8.0, NCBI, and Sol genomics network (Table 1). Wholesequence comparison of RTFLs was obtained from the Surveyed Conserved Motif Alignment Diagram and the Associating Dendrogram (SALAD) database (Mihara et al. 2009). SALAD is commonly used for genome-wide comparative analysis of annotated protein sequences in plants. The evolutionarily conserved short amino acid sequences in homologous proteins were identified as motifs using MEME software (http://meme.sdsc.edu/meme/intro.html) in SALAD. The motif significance is reported as the E-value upon MEME analysis, and the motif number in SALAD depends on this E-value. However, this numbering system sometimes results in dispersive motif numbers among the paralogs. In order to keep the motif numbers consecutive in the text, six motif numbers (Motif 5, 11, 12, $14,16,24)$ were slightly modified and re-numbered by considering a generality in distribution of each motif among various plant taxa (see the modification in Table S2). Annotations with similar motif patterns were grouped into the same clades according to the value of approximately

Table 1188 RTFL members used in the comparative analysis

\begin{tabular}{|c|c|c|c|}
\hline Species & Peptide abbreviation/Nomination & Database/Supple & Number of paralogs \\
\hline \multicolumn{4}{|l|}{ Liverwort } \\
\hline Marchantia polymorpha & MARPO & Prof. John L. Bowman & 1 \\
\hline \multicolumn{4}{|l|}{ Moss } \\
\hline Physcomitrella patens & PHYPA & SALAD $^{\mathrm{a}}$ & 2 \\
\hline \multicolumn{4}{|l|}{ Gymnosperm } \\
\hline Picea sitchensis & PICSI & Pfam $^{\text {b }}$ & 2 \\
\hline \multicolumn{4}{|l|}{ Monocotyledons } \\
\hline Oryza sativa & From RAP-DB ${ }^{c}$ & SALAD & 20 \\
\hline Brachypodium distachyon & BRADI & SALAD & 5 \\
\hline Sorghum bicolor & SORBI & SALAD & 4 \\
\hline Zea mays & GRMZM & SALAD & 10 \\
\hline Hordeum vulgare var. distichum & HORVD & Pfam & 3 \\
\hline \multicolumn{4}{|l|}{ Eudicotyledons } \\
\hline Ricinus communis & RICCO & Pfam & 8 \\
\hline Carica papaya & CARPA & SALAD & 2 \\
\hline Glycine $\max$ & GLYMA & SALAD & 40 \\
\hline Medicago truncatula & MEDTR & SALAD & 8 \\
\hline Populus trichocarpa & POPTR & SALAD & 14 \\
\hline Vitis vinifera & VITVI & SALAD & 2 \\
\hline Fragaria vesca & FRAVE & SALAD & 3 \\
\hline Arabidopsis thaliana & RTFL1-23/ROT4 & SALAD & 24 \\
\hline Arabidopsis lyrata subsp. lyrata & ARALL & Pfam & 17 \\
\hline Thellungiella halophila & THHALV & Pytozome v8.0 ${ }^{\mathrm{d}}$ & 7 \\
\hline Thellungiella parvula & THEPA & $\mathrm{NCBI}^{\mathrm{e}}$ & 5 \\
\hline Cleome spinosa & ROSI & Pfam & 2 \\
\hline Solanum lycopersicum & SOLYC & Sol genomics network ${ }^{\mathrm{f}}$ & 3 \\
\hline Aquilegia caerulea & AQUCA & Pytozome v8.0 & 6 \\
\hline Total & & & 188 \\
\hline
\end{tabular}

a http://salad.dna.affrc.go.jp/salad/en/

${ }^{\mathrm{b}}$ http://pfam.xfam.org

${ }^{c}$ http://rapdb.dna.affrc.go.jp

d http://www.phytozome.net

e http://www.ncbi.nlm.nih.gov

${ }^{\mathrm{f}} \mathrm{http}: / /$ solgenomics.net 
unbiased (AU) or bootstrap probability (BP) (Shimodaira 2002, 2004). Clustering was calculated using pvclust in $R$ software (http://www.r-project.org/).

Phylogenetic relationships between RTFLs in $O$. sativa and Arabidopsis were evaluated using MEGA version 6 (Tamura et al. 2013). A total of 43 RTF sequences of $O$. sativa and Arabidopsis were used for the analysis. Molecular Phylogenetic analysis was inferred using the maximum likelihood method based on the JTT matrix-based model (Jones et al. 1992). The bootstrap consensus tree inferred from 1000 replicates (Felsenstein 1985) was used to represent the evolutionary history of the taxa analyzed. Initial tree(s) for the heuristic search were obtained by applying the Neighbor-Joining method to a matrix of pairwise distances estimated using a JTT model.

\section{Anatomical analysis}

The first leaves of 25-day-old plants were collected and cleaned with a chloral hydrate solution $\left(4 \mathrm{~g} \mathrm{~mL}^{-1}\right.$ chloral hydrate and $0.4 \mathrm{~g} \mathrm{~mL}^{-1}$ glycerol) as described by Tsuge et al. (1996). Palisade cells were observed using the Leica upright materials microscope (DM2700 M; Leica, Wetzlar, Germany). To record root growth, 3 days after germination a total of 30 Arabidopsis individuals of the wild type, ROT4 $o / x$, and $O S R T F L 3 o / x$ were scanned every 2 days. Individual cell sizes and root lengths were measured as described by Narita et al. (2004) using the ImageJ 1.48 program (National Institutes of Health; http://imagej.nih.gov/ij/).

\section{Results}

Comparative analysis of the RTFL family

The RTFL family is widely conserved among land plants and shares no sequence similarities with identified proteins or well-characterized motifs (Narita et al. 2004; Wen et al. 2004). Therefore, we investigated the biological functions of RTFL orthologs to increase our understanding of their effects on the control of plant organogenesis. SALAD is a motif-based database for plant comparative proteomics. This program can be used to predict biological function based on the hypothesis that proteins with similar motifs have similar biochemical properties and thus related biological functions (Mihara et al. 2009). Consequently, we collected 188 RTFLs among 22 species with full-length amino acid sequences for comparative analysis using SALAD (Table 1, see the whole amino acid sequences of 188 RTFLs in Supplemental Table S1). A total of 8 RTFLs were excluded by SALAD due to the low similarity calculated using the MEME software (Bailey et al. 2009), and the remaining 180 RTFLs were shown in the comparative analysis (Fig. 1, see the complete tree in Fig. S1). These sequences cover a wide range of land plant lineages, including liverworts, moss, gymnosperms, and angiosperms.

A total of 73 motifs were identified using the MEME suite (Bailey et al. 2009) among 180 RTFLs (see all motif sequences in Supplemental Table S2; see the nomination rules of motifs in the "Materials and Methods"). The RTF domain, which was used for blasting RTFL members, was presented as Motif 1 among all RTFLs (Figs. 1, S1). The $\mathrm{N}$-terminal region of the RTFL family is less conserved among RTFL members, and no predictable signal peptides have been identified (Narita et al. 2004; Wen et al. 2004). This was confirmed by our results since the remaining 72 variable motifs (Motifs 2-73) were mostly identified around the N-terminus without any signal motifs (Figs. 1, S1).

The 180 RTFLs were grouped into four clades (Clades 1-4; Fig. 1) based on the motif patterns. Conserved motifs could be found in an interspecific manner. Excluding Motif 1 that defined the RTFL family, Motifs 2-12 were found in various species from liverworts to angiosperms (Figs. 1, S1). In Clade 4, Motifs 9, 19, 59 and 71 were found in Marchantia polymorpha (liverwort), Physcomitrella patens (moss), and Picea sitchensis (gymnosperm) (Figs. 1d, blue, green and yellow-green arrows, S1, respectively). Motifs 2 and 3 were found among RTFLs of eudicots and monocots in Clade 2 and Clade 3 (Figs. 1b, c, S1). In addition, Motifs 2-4 and 7-9 were found among species in Clade 2, which consisted of only eudicots (Figs. 1b, S1). Clade 1 consisted of two long RTFLs with diverse motifs (Figs. 1e, S1). Conserved motifs could also be found within specific families. Two types of motif combinations (Motifs 3, 4 and 8; Motifs 4, 7, and 9) were specific in Brassicaceae, and were also observed in Arabidopsis thaliana, A. lyrata subsp. lyrata, Thellungiella parvula, and T. halophila (Figs. 1b, S1). Motifs 2-4 and 8 were conserved among RTFLs in Glycine $\max$ and M. truncatula of Leguminosae (Figs. 1b, c, S1). Motifs 2, 3, and 10 were conserved among all species of Gramineae, which we compared to O. sativa, Brachypodium distachyon, Sorghum bicolor, Zea mays, and Hordeum vulgare var. distichum (Figs. 1c, S1). According to the motif patterns, RTFL members in Arabidopsis could be divided into five subgroups (Table 2); Subgroup 1 contained Motifs 1, 3 and 4, which were shared by RTFL 2 and 3 in Clade 2 (Figs. 1b, S1); RTFL4 and 5 in Clade 2 (Figs. 1b, S1) were grouped into Subgroup 2, both of which contained Motif 1, 4, 7 and 9; Subgroup 3 included ROT4, RTFL1 and RTFL 7-11 in Clade 3 (Figs. 1c, S1), with a pattern of Motifs 1 and 2 in common; RTFL15-19 and 21 in Clade 4 (Figs. 1d, S1) were grouped into Subgroup 4, with Motifs 1 and 6 in common; the remaining RTFLs 

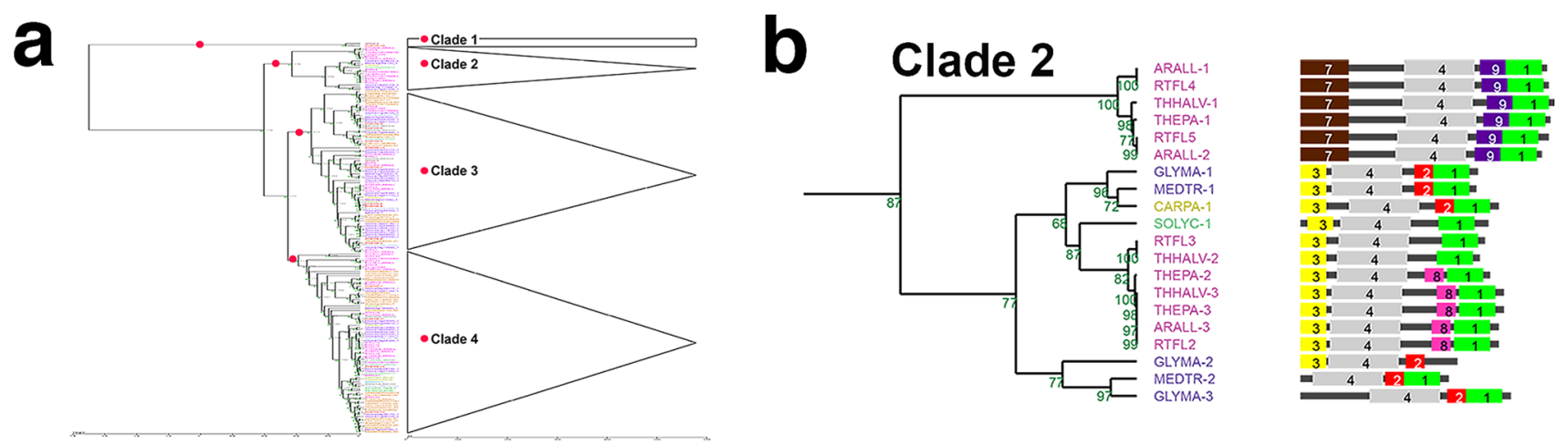

C
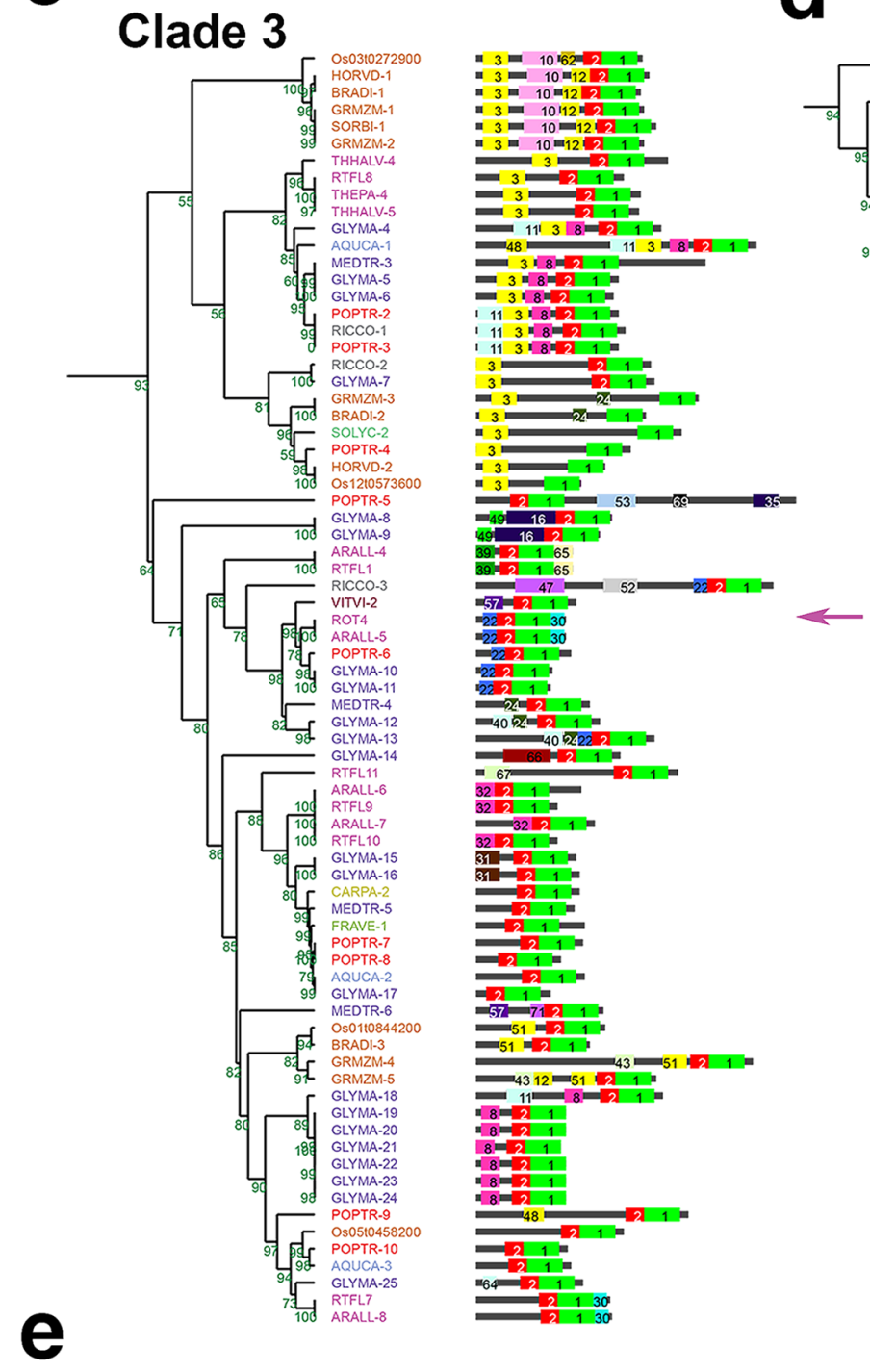

d Clade 4

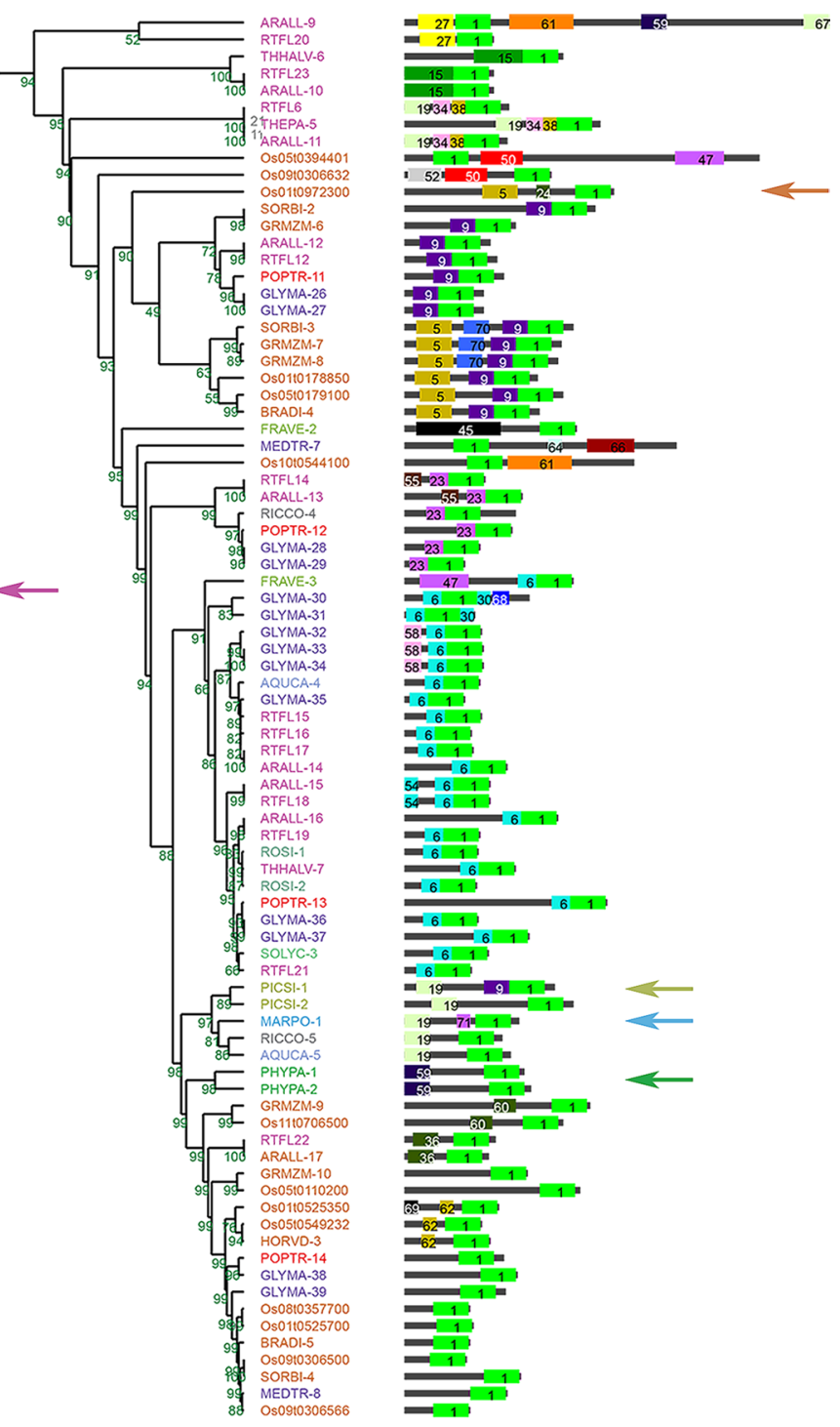

Clade 1
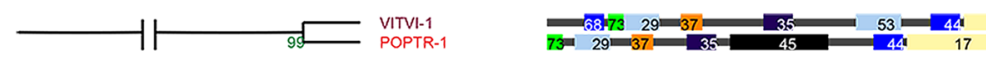

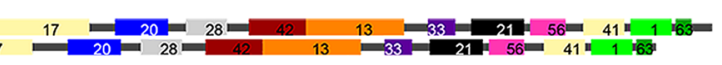


4Fig. 1 Comparative analysis of the 180 RTFL family among land plants. The whole tree (a) was generated using SALAD (Mihara et al. 2009) and divided into four clades (b-e) based on the bootstrap values, and was approximately unbiased. Only bootstrap values were shown beside the branches (in green). Full amino acid sequences of RTFLs were used for analysis. The complete tree is shown in Fig. S1. c Arrow indicates ROT4. d Arrows (from top to bottom) indicate RTFLs of Oryza sativa (OsRTFL3), Picea sitchensis, Marchantia polymorpha, and Physcomitrella patens, respectively. RTFLs of species from one family were marked with the same color in the figure. Motifs were numbered in different colors in the boxes. Green numbers around the branches indicated the bootstrap values

(RTFL 6, 12, 13, 14, 20, 22 and 23) in Clade 4 (Figs. 1d, S1) showed diverse motif patterns with only Motif $1 /$ functional RTF domain in common, and thus were grouped into Subgroup 5 (RTFL13 was excluded by SALAD in Figs. 1, $\mathrm{S} 1$, but was included in the same clade with RTFL14 when analyzed with lower amounts of RTFLs, unpublished data).
Phylogenetic analysis of RTFL members in Arabidopsis and $O$. sativa

Based on the above comparative analysis, we examined RTFL diversity between Arabidopsis (an eudicot) and $O$. sativa (a monocot), both of which are common model plants that have been fully sequenced (The Arabidopsis Genome Initiative 2000; mads Genome Sequencing Project 2005). A total of $90 \%$ of Arabidopsis genes are believed to have homologs in the rice genome (International Rice Genome Sequencing Project 2005), and here 20 RTFL orthologous members in $O$. sativa exhibited diverse motif patterns (Table 3; Figs. 1, S1). Therefore, we generated a phylogenetic tree of 43 RTFL members from Arabidopsis and $O$. sativa (Fig. 2a) based on the conserved RTF sequences (identified as Motif 1 in Figs. 1, S1) for two reasons: (a) RTF domain/Motif 1 of Arabidopsis is sufficient to induce the RTFL-overexpression phenotypes in leaves and fruits
Table 2 Subgroups of 24 RTFL members in Arabidopsis
Circles represent the related motifs found in the corresponding RTFL members Only the motifs shared by all members in a subgroup were shown in the table

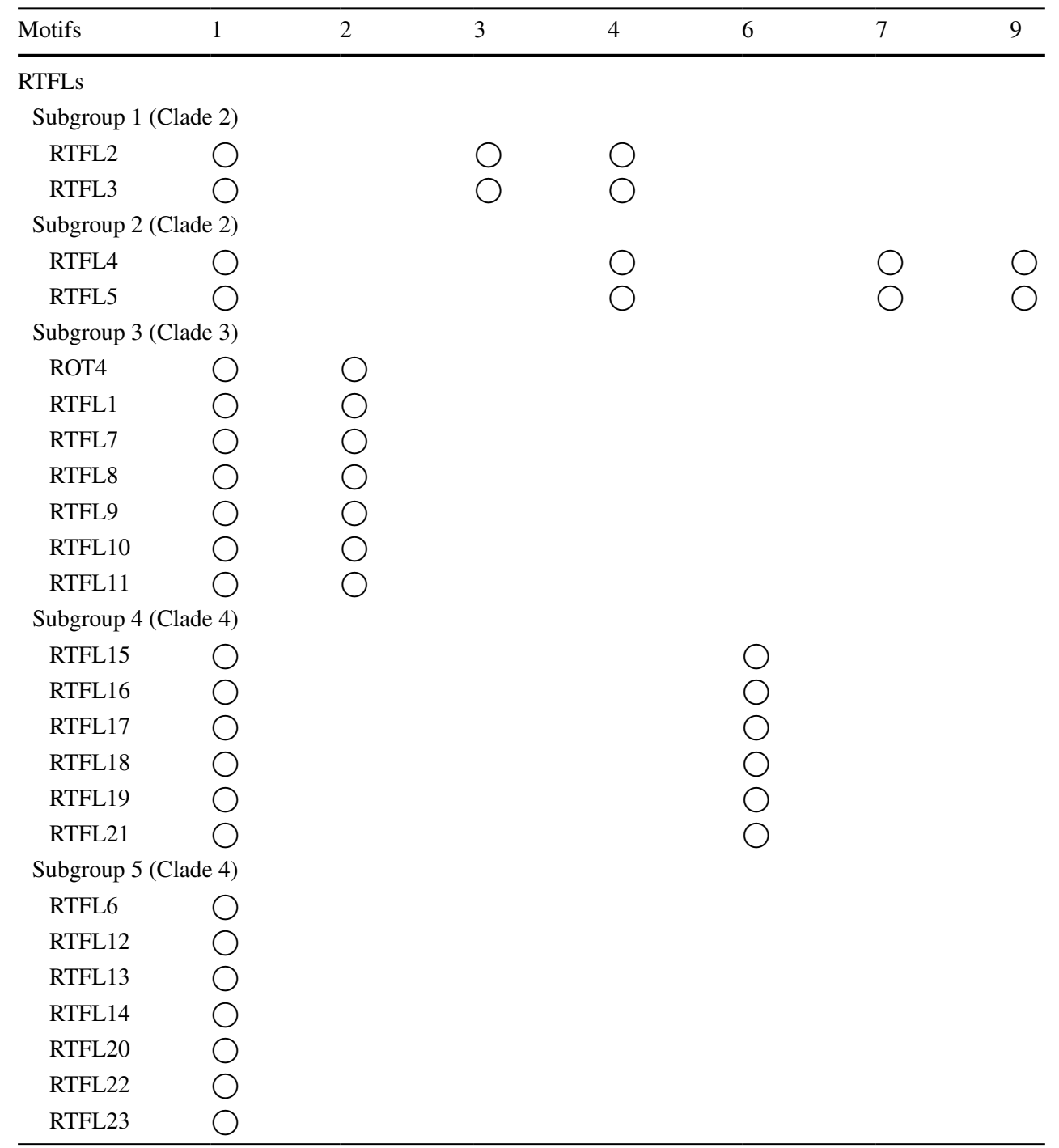


Table 3 Species of RTFLs which exhibit similar motif patterns as ROT4, DVL1-5 and OsRTFL3

\begin{tabular}{lll}
\hline Species of RTFLs & $\begin{array}{l}\text { Paralog } \\
\text { numbers }\end{array}$ & $\begin{array}{l}\text { Published RTFLs with } \\
\text { similar motif patterns }\end{array}$ \\
\hline Vitis vinifera & 2 & ROT4 \\
Ricinus communis & 6 & ROT4 \\
Sorghum bicolor & 4 & OsRTFL3 \\
Brachypodium distachyon & 5 & OsRTFL3 \\
Fragaria vesca & 3 & DVL1-5 \\
Aquilegia caerulea & 6 & DVL1-5 \\
Solanum lycopersicum & 3 & DVL1-5 \\
Cleome spinosa & 2 & DVL1-5 \\
\hline
\end{tabular}

(Ikeuchi et al. 2010); (b) RTF domain/Motif 1 is the only sequence/motif conserved among all RTFLs in Arabidopsis and $O$. sativa (Figs. 1, S1). The short length of RTF domains resulted in weak bootstrap values (data not shown), but the general topological relationships were observed regardless of the analysis parameters. Os01t0972300 was phylogenetically clustered into the same clade with ROT4 (Fig. 2a; arrows) based on RTF sequences/Motif 1, although they were in different clades based on the comparative analysis of whole amino acids sequences/whole motif patterns (Fig. 1c, d, purple and orange arrows). Os01t0972300 encodes 124 amino acids and was named OsRTFL3 in this report, which follows the nomination of OsRTFL1 and OsRTFL2 in Narita et al. (2004).

OsRTFL3 has similar functions as ROT4 in the development of above-ground organs

The long evolutionary history and conserved sequence of RTFL peptides are indicative of their essential functions in land plant evolution. To investigate whether the RTFL family is functionally conserved between eudicots and monocots, OsRTFL3 was constructed under the $35 \mathrm{~S}$ promoter of the Cauliflower mosaic virus (CaMV35S) and transformed into wild-type Arabidopsis (Col-0). We established

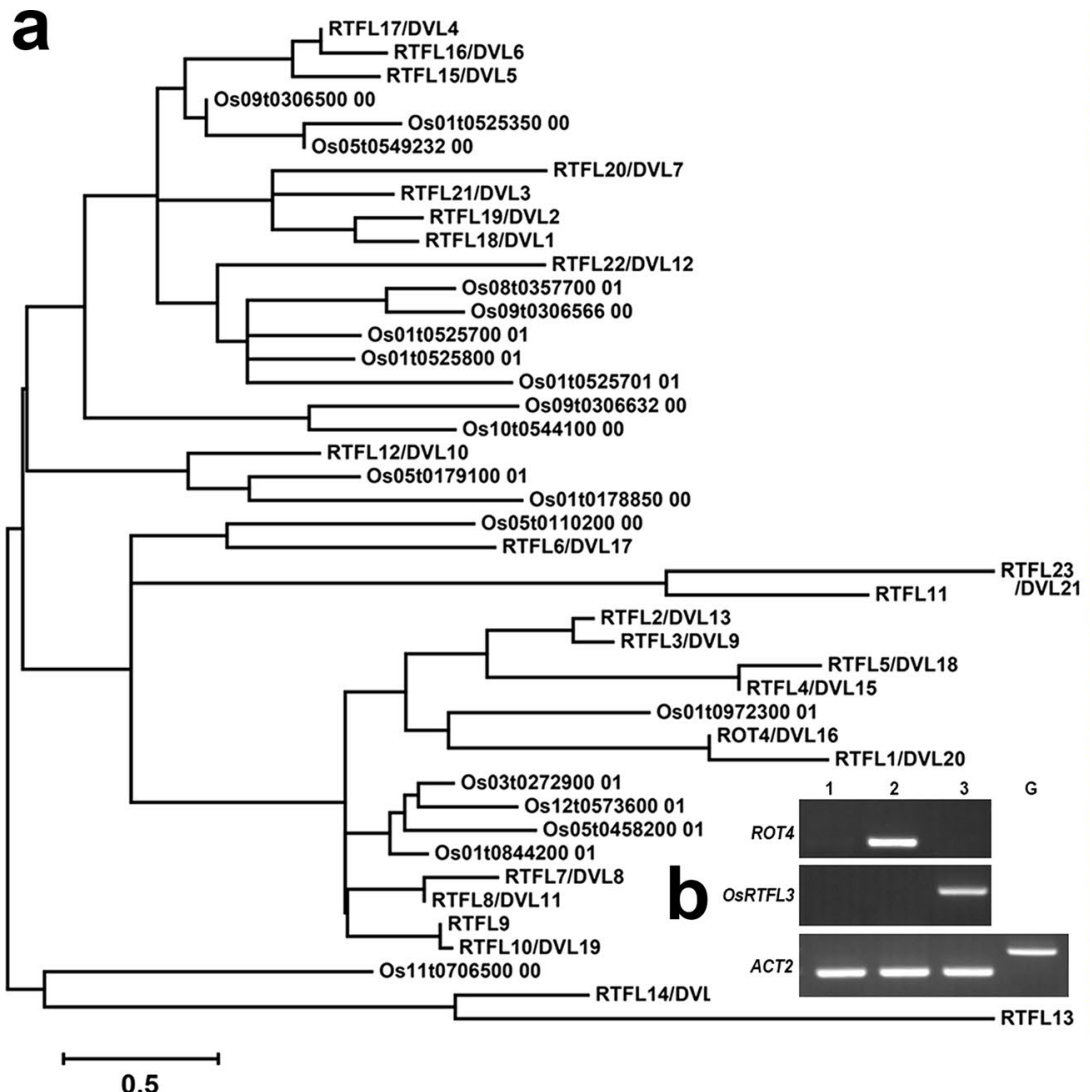

KGLGAVLKEQRAKLY I I RRCV VMLLCWHD 29 RGLGGVLREQRAKLY I I KRCVVMLLCWQD 29 RGVGGVVREQKAKLY I I RRCVVMLLCWHD 29 GKLQNV LREQKARLY I I RRCV VMLLCWSD 29 GG I SKVVREHKARLY I I RRCV VMLLCWHD 29 GGLSRMLREQKARLY I I RRCV VMLLCYHD 29 KGVGEFLKKKKGRFY I I GKC I TMLLCSHK 29 KKLEY KKLGY RRLGKYLKEQKGRI YIIRRCVMMLLCSHD 29 RRLGKYMKEQKGRIYIIRRCMVMLLCSHD 29 LTPNRSLKETRSRLY I I RRCLVMLLCWRE 29 GRLNKALKEKRAKLY I I RRCV VMLLRWSD 29 GRMTRA LKEHRARLY I I RRC I VMLLCWHE 29 GGFGRA I KQQRARLY I I QRCV VMLLRWQD 29 RG I ARA LKEHKARLY I I RRCV VMLLRC- - 27 GGFSKSLKEQRSRLY I I SRCV VMLLRWHD 29 GRLNRAFREKRARFY I FRRCV IMLLRWSD 29 WLLAAAREORFY WLLQAAAREQRSRFY DRCLLMAKQQRTRLYILRRCV SMLLCWHD 29 RRCYAVLKQQRTRLYILRRCV SMLLCWHE 29 GRCNAV LKEHKTRLY I LGRCV SMLLCWHN 29 HRLTKT VKEHRARFY I I RRC I QML I CWRD 29 KGCLAMVKERRSRFY I ARRC I LMLLCWHK 29 EKMKRT VRQQRAKLH I I R I C I TMLLSSDD 29 SKCT SLMKQQHARLCI I RLCATMLLRSYT 29 QKY SSLAKEQKARFY I MRRCVAMLVCWHK 29 QKYSSLAKEQKGRFY I MRRCVAMLVCWHK 29 QK GKCFKVAKEHKSRFY IKR GKCFKVAKEHKSRFYIIKRCVLMLVCWHK 29 KKCVEAVKEHRARFY I VRRCVSMLVCWRD 29 QKCSHVVKKQRAKFY I LRRC I AML V CWHD 29 AKC SHMVRKQRAKFY I LGRCLAMLVCGRG 29 SRCAGLVKEQRARFY I MRRCV TMLVCWKD 29 GRLASLVKEQRARFY I MRRCVTMLVCWRD 29 RRCARLVKEQRARLY I VRRC I TMLACWRD 29 CRCVRLVKEQRARFY IMRRCVTMLVCWHE 29 RKCGRLVKEORARFY IMRRCVVML I CWTD 29 RKCARLVKEQRARYY IMRR VIML CWTD 29 RKCARLVKEQRARFY MARCVIML CWRD 29 SKCASLVKEQRARLYILRRCATMLCCWYI 29 SKCVSLVKEQRARLYILRRCATMLCCWYI 29 RSVSGRVREQRARLY I MRRCV SLL I SST F 29 KRCSKQ I KEQRARLY I I WKCAVFLLSSHD 29 LRCWDWCKEQRTRAY I I WRCL I FLLRWDD 29
Fig. 2 Molecular phylogenetic analysis of RTFL members. a Phylogenetic analysis and alignment of 43 RTFL members from Oryza sativa and Arabidopsis. RTF domains, which show homology at the C-terminus of the RTFL family, were aligned using the ClustalW software and constructed using MEGA 6.0 using the maximum-likelihood method. Arrows indicate Os01t0972300_01 (upper) and ROT4 (below). b RT-PCR analysis of ROT4 and OsRTFL3 mRNA accumulation. Total RNAs of mature 10-day-old whole plants were used. Lane 1 , wild type; lane 2, ROT4 o/ $x$; lane 3 , OsRTFL3 o/ $x$. ACT2 was used as an internal control. Lane $G$ shows a negative control using genomic DNA of 10-day-old wild-type plants 
five independent transgenic lines and selected one line for further study after confirming that all individuals showed fundamentally similar phenotypes. Transgenic plants overexpressing ROT4 were used in Narita et al. (2004), with the coding sequence of ROT4 constructed under CaMV35S. The high expression level of ROT4 and OSRTFL3 was confirmed using RT-PCR in the transgenic plants (Fig. 2b). No detectable amplification of ROT4 was observed in the wildtype Arabidopsis under our PCR conditions, which could be explained by the low expression level. These two overexpressing lines were termed ROT4 o/ $x$ and OsRTFL3 $o / x$ in the following content.

We next compared the morphology of wild-type plants, ROT4 $o / x$, and OsRTFL3 $o / x$. Both ROT4 $o / x$ and OsRTFL3 $o / x$ showed a pronounced reduction in organ size (Fig. 3a, b). The reduction in blade area, petiole length, blade length, and width of OsRTFL3 $o / x$ was more significant when compared with wild type and ROT4 $o / x$ (Figs. 3c,4a, b; $P<0.001$, paired student's t test). OsRTFL3 o/x also showed a short-organ phenotype in inflorescences and fruits, similar to ROT4 $o / x$ (Fig. $3 \mathrm{~d}$, e). In addition, fruits of OsRTFL3 o/ $x$ were wider than those of wild type and ROT4 $o / x$ (Fig. 3e). Although filaments and stamens were much shorter in OsRTFL3 $o / x$, they could reach the stigma at later developmental stages. Therefore, OsRTFL3 o/x was fully fertile, as was ROT4 $o / x$ (Narita et al. 2004). The above comparison of gross morphology demonstrated that phenotypes of OsRTFL3 $\mathrm{o} / x$ in shoots were similar to ROT4 $o / x$, but were quantitatively different.

Organ size is determined by both cell size and number (Tsukaya 2006). To examine whether the reduced leaf size of ROT4 o/ $x$ and OsRTFL3 $\mathrm{o} / x$ were induced by a decrease in cell number and/or size, the number and size of palisade cells in the first rosette leaves of wild-type plants, ROT4 $o / x$, and OsRTFL3 $o / x$ were measured. The total number of palisade cells per leaf blade in both ROT4 $o / x$ and OsRTFL3 $o / x$ decreased significantly, with a more severe reduction in OsRTFL3 $\mathrm{o} / x$ (Fig. 4c). To confirm whether the decreased cell number was related to the effect of ROT4 $o / x$ and OsRTFL3 $o / x$ on leaf shape, the palisade cell numbers in both the leaf-length and leaf-width direction were counted. The results showed that the cell number of OsRTFL3 $\mathrm{o} / \mathrm{x}$ and ROT4 $\mathrm{o} / \mathrm{x}$ in the leaf-length direction decreased in a similar pattern as the decrease in total cell number in the subepidermal layer. However, cell numbers along the leaf-width direction in OsRTFL3 $\mathrm{o} / x$ significantly decreased compared with wild type and ROT4 $o / x$ (Fig. $4 \mathrm{~d}$ ). Similarly, the size of palisade cells in both ROT4 $o / x$ and

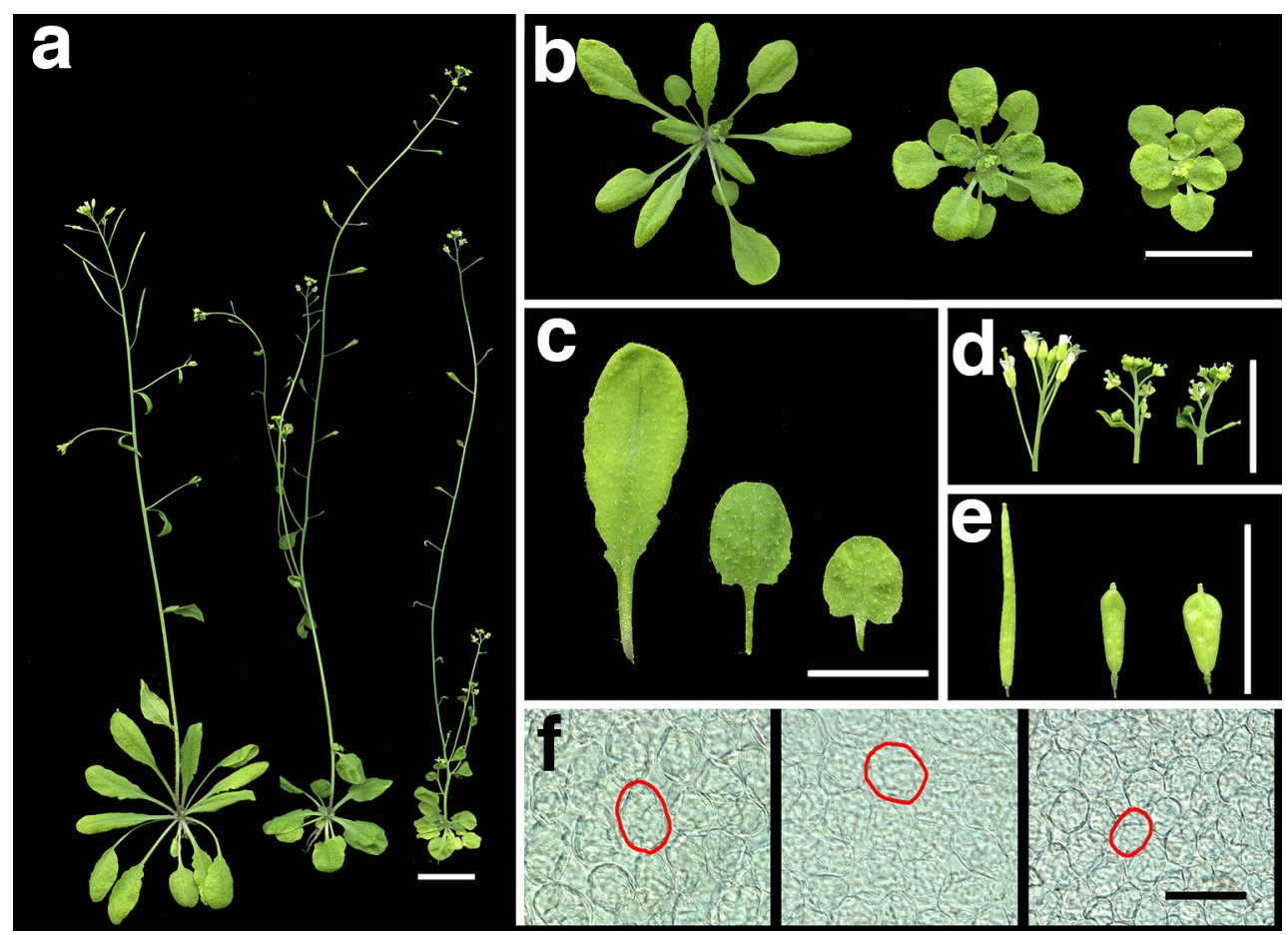

Fig. 3 Phenotypes of OSRTFL3 $o / x$. a Thirty-five-day-old plants of wild-type Arabidopsis, ROT4 $\mathrm{o} / \mathrm{x}$, and $O s R T F L 3 \mathrm{o} / \mathrm{x}$ (from left to right). b Twenty-five-day-old rosette leaves of the wild type, ROT4 $o / x$, and OsRTFL3 $o / x$ (from left to right). c The fifth rosette leaves of the wild type, ROT4 $o / x$, and OsRTFL3 $o / x$ (from left to right). d Inflorescences of the wild type, ROT4 o/x, and OsRTFL3 $\mathrm{o} / \mathrm{x}$ (from left to right) plants. e Fruits of 35-day-old wild type, ROT4 $o / x$, and OSRTFL3 $o / x$ (from left to right). f Palisade cells in the middle portion of first foliage leaves of 25-day-old wild type, ROT4 $\mathrm{o} / \mathrm{x}$, and OSRTFL3 $\mathrm{o} / \mathrm{x}$ (from left to right) plants. Red circles indicate a single palisade cell. Scale bars $=1 \mathrm{~cm}(\mathbf{a}-\mathbf{e}) ; 100 \mu \mathrm{m}(\mathbf{f})$ 

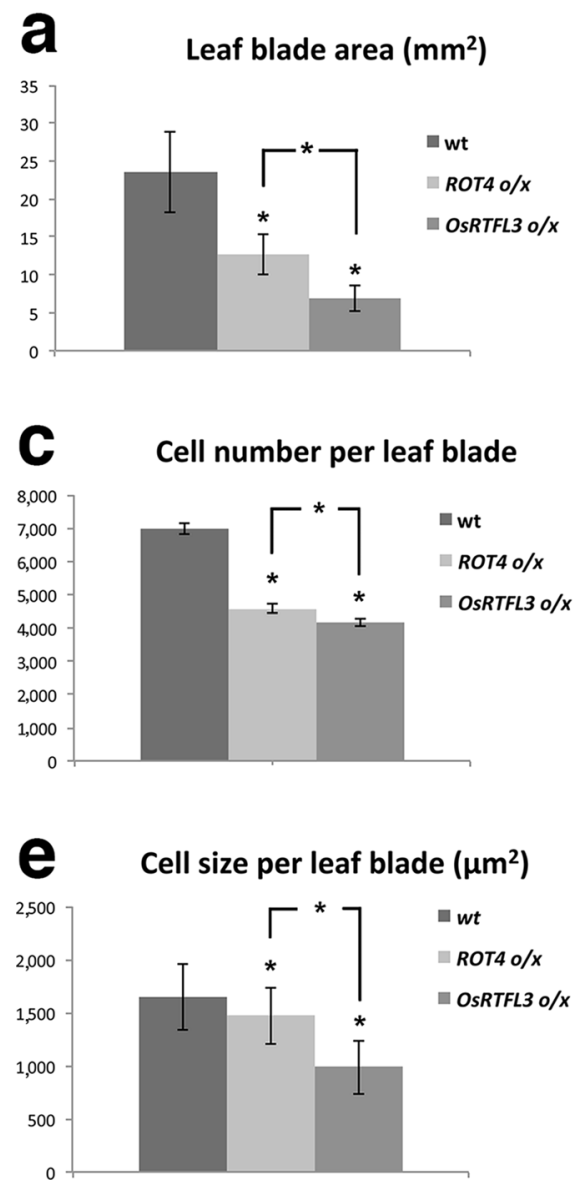

Fig. 4 Measurement of leaf morphological characteristics in ROT4 $o / x$ and OsRTFL3 $o / x$ a The blade area of 25-day-old wild type (left), ROT4 o/x (middle), and OsRTFL3o/x (right). $n=10$. Values represent the mean $\pm \mathrm{SD}$. b Leaf petiole, blade length, and width of first leaves of 25-day-old wild type (left), ROT4 o/x (middle), and OsRTFL3 $\mathrm{o} / x$ (right) were measured using ImageJ 1.29 program $n=10$. Values represent the mean \pm SD. c, $\mathbf{d}$ Numbers of palisade cells of the first leaf blade of 25-old-day plants. c Numbers of palisade cells in the subepidermal layer per leaf blade; $\mathbf{d}$ Numbers of

OSRTFL3 $o / x$ significantly decreased, with a more severe reduction in OsRTFL3 $o / x$ (Fig. 4e). This pattern indicates that both ROT4 o/x and OSRTFL3 control polar cell proliferation as well as cell expansion in the lateral organs, suggesting that OsRTFL3 has a similar function as ROT4 in the control of organogenesis when overexpressed in Arabidopsis. In addition, OsRTFL3 showed a unique function in negatively regulating the cell number along the leaf-width axis when overexpressed, which was not observed in ROT4 $o / x$ lines.

\section{OsRTFL3 o/x inhibited root growth}

Although at least six RTFL members in Arabidopsis have been overexpressed and resulted in the dominant "roundleaf" phenotype, no significant differences were observed
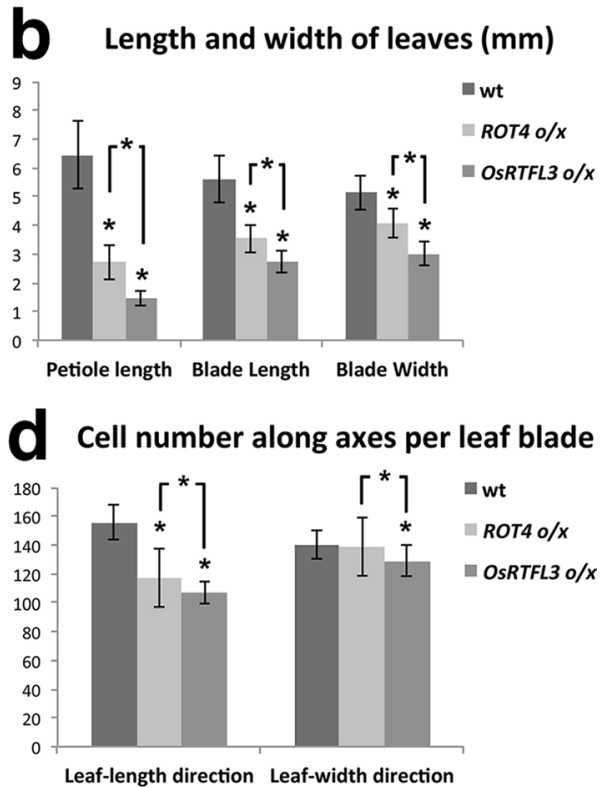

palisade cells in leaf-length and leaf-width directions. The columns: wild type (left), ROT4 o/x (middle), and OsRTFL3 o/x (right) $n=10$. Values represent the mean \pm SD. e Palisade cell size of the first leaf blade of 25-old-day plants. Cell area of 10 cells was measured for each line. Columns indicate palisade cell size of the wild type (left), ROT4 o/x (middle), and OsRTFL3 $o / x$ (right) $n=10$. Values represent the mean $\pm \mathrm{SD}$. Asterisk indicates significant differences calculated using Student's $t$ test $(P<0.001)$

in morphological features of roots between wild type and RTFL overexpressors (Narita et al. 2004; Wen et al. 2004). However, according to our observations, both ROT4 o/x and $O S R T F L 3 \mathrm{o} / \mathrm{x}$ generated shorter primary roots, and OSRTFL3 $o / x$ exhibited a more severe phenotype (Fig. 5a). The rate of root elongation decreased severely in OSRTFL3 $o / x$ based on the time-course analysis, which was also observed in ROT4 o/x, but more mildly. (Fig. 5b). In addition, the roots of $O s R T F L 3 o / x$ almost stopped elongating around the fifth day after germination, while the elongation rate began to accelerate in the wild type and ROT4 o/x. Inhibition was observed in another three independent lines of OsRTFL $3 o / x$, and all OsRTFL3 $o / x$ lines had a capability of generating lateral roots (Figs. 5a, S2). The developmental defects in root growth of both ROT4 o/ $x$ and OsRTFL3 $o / x$ were inconsistent with the previous RTFL-overexpressing 
Fig. 5 Root growth of 10-dayold wild type (left), ROT4 o/x (middle), and OsRTFL3 $\mathrm{o} / \mathrm{x}$ (right) plants. a Root phenotype of 10-day-old plants. b Timecourse analysis of root length $n=30$, Scale bars $=1 \mathrm{~cm}$

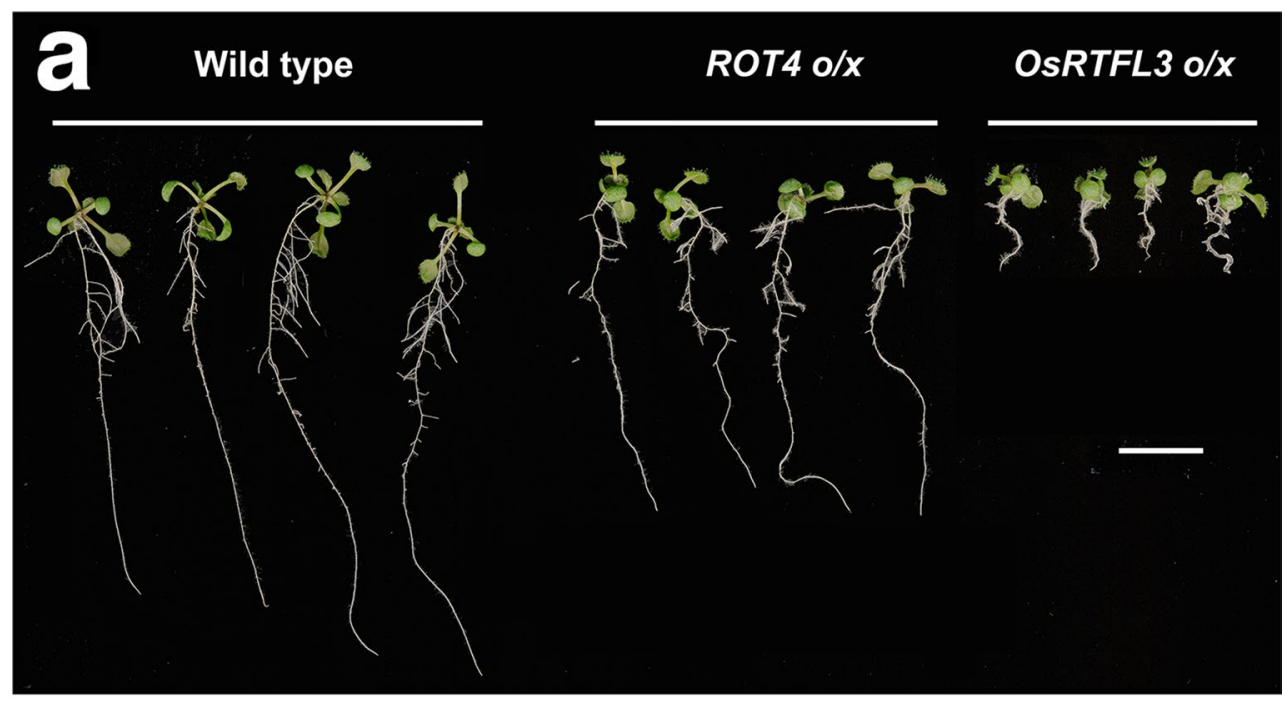

b

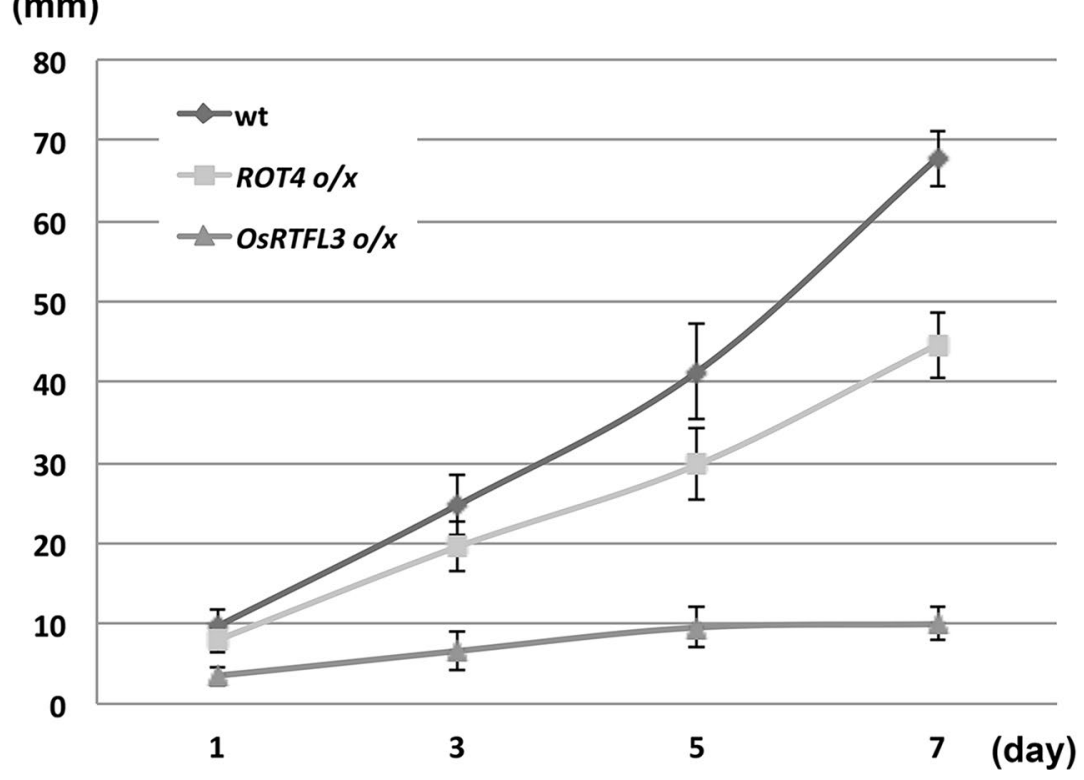

phenotypes observed in Arabidopsis. The phenotypes of $O S R T F L 3 \mathrm{o} / x$ regarding the regulation of cell numbers along the leaf-width axis and root growth suggested that OsRTFL3 $o / x$ may have unique functions in the control of organogenesis, in addition to the common functions as ROT4.

\section{Discussion}

Diversity of the N-terminal structure among RTFLs

All RTFLs share a highly conserved domain in the C-terminus (RTF domain) and diverse sequences in the N-terminus. Examining the diversity of the N-terminal structure is one application of comparative RTFL analysis.
Changes in motif architecture preferentially occur at the protein termini since they are more tolerant to domain insertions or deletions due to terminal flexibility (Bjorklund et al. 2005; Buljan and Bateman 2009; Weiner et al. 2006). This domain shaping may also occur in the ancestral sequence of the RTFLs. The domains/motifs of the RTFL family are quite short, ranging from 6 to 35 amino acids (examples are shown in Table S2). Modulation of the RTFL motifs may not incur large alternations at the gene level compared with other "normal-size" proteins with an average length of 120 (Buljan and Bateman 2009). Thus, the RTFL peptide family may be more tolerant to domain changes due to the small sequence sizes, which may explain the diverse structure of the $\mathrm{N}$-terminus in the RTFL family. 
Motif patterns of RTFL members

This is the first study to perform a comparative analysis of the RTFL family. In total, 180 RTFL members from 22 species were grouped into four clades (Clades 1-4 from top to bottom of the tree; see Fig. 1b-e). RTFLs of Marchantia polymorpha, Physcomitrella patens, and Picea sitchensis, which share an early evolutionary position among land plants, were clustered into Clade 4 (Fig. 1d, green and yellow-green arrows), indicating that the motif patterns in Clade 4 may represent the ancestral structure of the RTFL family. Clade 3 (Fig. 1c) exhibited diverse motif patterns, and members in this clade were found in all flowering plants examined, including Arabidopsis (a dicot) and Oryza sativa (a monocot). This suggested that an evolutionary event occurred in the RTFL family during the initiation of flowering plants, and that the RTFL family may have gained additional motifs after divergence from its ancestors. The majority of RTFLs in Clade 3 contain Motif 2, indicating that the formation of Motif 2 was associated with the basic function of Motif 1 in the RTFL family among flowering plants (Fig. 1c). RTFL members in Clade 2 (Fig. 1b) shared uniform motif patterns and were observed only in eudicots. Excluding functional Motif 1, Motif 4 was also found among the RTFLs in Clade 2, suggestive of its specific role in the evolutionary process of eudicots (Fig. 1b). Meanwhile, all RTFLs of Gramineae (monocots) in Clade 3 contained motifs 2,3 , and 10, suggesting that these motifs play specific roles in monocot evolution (Fig. 1c). Based on the motif patterns, the RTFL family may have originated from early bryophytes and experienced an evolutionary event during the transition to flowering plants. The required new motifs, which were formed after the transition, may have different roles in the evolution of flowering plants and some specific families.

Functions of motifs in the RTFL family

The primary purpose of the SALAD program is biological and biochemical prediction. Proteins with similar motifs/ motif patterns are assumed to have related biological functions (Mihara et al. 2009). Wen et al. (2004) overexpressed DVL1/RTFL18, DVL2/RTFL19, DVL3/RTFL21，DVL4/ RTFL17, and DVL5/RTFL15 in Arabidopsis, which exhibited similar phenotypes, suggestive of their similar biological functions. These DVLs/RTFLs were clustered into the same subgroup based on motif patterns (Table 2, Subgroup 4), which supported the accuracy of the SALAD program in the aspect of biological and biochemical prediction in our study. Thus, RTFL candidates clustered closely to the reported RTFL members (ROT4/DVL16, DVL1/RTFL18, DVL2/RTFL19，DVL3/RTFL21，DVL4/RTFL17，DVL5/ RTFL15, and OsRTFL3) in the comparative analysis should be further explored; namely, VITVI-2 of Vitis vinifera, RICCO-3 of Ricinus communis (two motif patterns similar to ROT4, Fig. 1c), SORBI-2 and SORBI-3 of Sorghum bicolor, BRADI-4 of Brachypodium distachyon (grouped into the same clade with OsRTFL3, Fig. 1d), FRAVE-3 of Fragaria vesca, AQUCA-4 of Aquilegia caerulea, SOLYC-3 of Solanum lycopersicum, and ROSI-1 and ROSI-2 of Cleome spinosa (motif patterns similar to DVL1-DVL5, Fig. 1d). The biological function of the above 10 candidate members are thought to exhibit similar motif patterns as the reported RTFL members in Arabidopsis and $O$. sativa, which negatively regulate cell proliferation in the polar direction. Meanwhile, the numbers of RTFL paralogs in the above 10 candidate species are quite small, ranging from two ( $V$. vinifera) to six $(R$. communis) (Tables 1, 3). Further genetic studies on the above 10 candidate members, especially the construction of loss-of-function mutants, may increase our understanding of the exact function of the RTFL family since our current knowledge is limited due to the high gene redundancy of RTFLs in both Arabidopsis and O. sativa. In addition, genome information on the above candidates can be found on the related web sites.

Wen et al. (2004) reported that a wide range of phenotypic variation, (besides the common "short-leaf" phenotype), especially in fruits, was observed among the transgenic lines overexpressing DVL1-DVL5. Ikeuchi et al. (2010) demonstrated that overexpression of the core functional RTF region of ROT4 (Motif 1 in our study) is sufficient to induce the fruit phenotype. Thus, the remaining non-conserved sequences or motifs are responsible for the phenotype variation in fruits. Indeed, we previously found that two truncation lines overexpressing the ROT4 functional RTF domain with a deletion of non-conserved sequences in the $\mathrm{N}$-terminus and $\mathrm{C}$-terminus were similar to full-length ROT4 o/x, exhibiting the "short-leaf" phenotype, but showed a variation in the fruit phenotype (Narita et al. 2004). Similarly, Ikeuchi et al. (2010) also observed variations in fruit shape, and the frequency of the protrusion of inflorescence stems varied among a series of truncations when ROT4 was overexpressed (Ikeuchi et al. unpublished observation), supporting the above hypothesis. Hence, we examined OSRTFL3 from rice and demonstrated that the "sub-phenotypes" of OsRTFL3 o/x differed in fruit shape, cell number, and root development, while the common "short-leaf" phenotype was observed. According to our motif alignment (Fig. 1), no common motifs exist among ROT4, DVL1-DVL5, or OsRTFL3, excluding the functional Motif 1 (Fig. 6). Since the Motif 1/RTF domain is functional in the RTFL family and induces dominant phenotypes, all RTFL overexpressors generate similar "shortleaf" phenotypes (Ikeuchi et al. 2010). However, the "subphenotypes" vary among samples, which suggests that 
DVL1/ RTFL15

DVL2/ RTFL17

DVL3/ RTFL18

DVL4/ RTFL19

DVL5/ RTFL21

ROT4

OsRTFL3

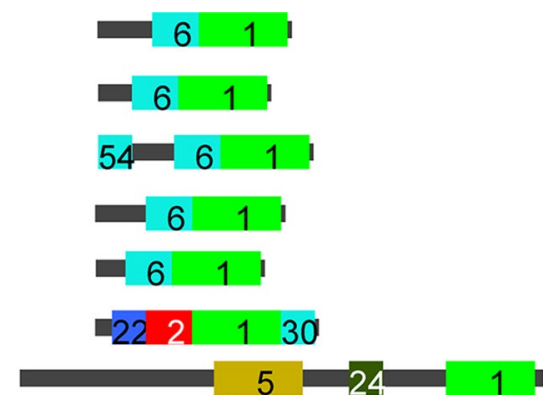

Fig. 6 Motif patterns of DVL1-5, ROT4, and OsRTFL3 (Modified from Figs. 1, S1)

the core RTF region (Motif 1) may be responsible for the "short-leaf" phenotype and that N-terminal motifs or the non-conserved sequences in RTFL members may perform specific functions contributing to the variable phenotypes. The possibility remains that such phenotypic variations can be caused by positional effects, namely, the fluctuating expression levels among transgenic plants. However, an examination of a series of comparisons among independent lines does not support this hypothesis.

Perspectives on the study of the RTFL family

The high level of gene redundancy hinders studies on the RTFL family. Without analyzing loss-of-function mutants, we could not thoroughly examine RTFL function. Based on our results, species with a lower number of paralogs carrying similar motif patterns as the reported RTFLs could be used to establish and study the loss-of-function lines. In addition, we observed a lower level of RTFL copies in bryophytes: one RTFL in M. polymorpha and two RTFLs in P. patens. Thus, studies on the RTFL family in bryophytes can increase our understanding of the function and evolution of RTFLs compared with other seed plants.

In this study, we identified subfunctions of motifs in the RTFL family. Future studies should explore the truncation or addition of specific motifs/motif patterns to characterize motif subfunctions. Meanwhile, the effects of representative orthologous RTFLs with similar motif patterns as the reported RTFLs should be examined based on overexpression. Details on the cell size and number, as well as anatomy in different root zones, should be confirmed in ROT4 $o / x$ and $O s R T F L 3 o / x$. In addition, plant hormones play an important role in the regulation of root growth. Studies on hormones such as auxin and cytokinins also reported abnormal root phenotypes with shorter or longer roots (Petricka et al. 2012). The phenotypes of cellular structure coupled with related candidate hormones should be examined to increase our understanding of root phenotypes in the above two RTFL overexpressors.
Acknowledgments We thank Prof. John L. Bowman (Monash University, Australia) for the RTFL sequence in Marchantia polymorpha, Prof. Hiroyuki Hirano (The University of Tokyo) for supplying us with T65 plants. This work was supported by the Japan Society for the Promotion of Science (Grants-in-Aid for Creative Scientific Research and Scientific Research A), the Ministry of Education, Culture, Sports, Science and Technology, Japan (Scientific Research on Priority Areas and Scientific Research on Innovative Areas), and the Mitsubishi Foundation.

Conflicts of interest The authors declare that they have no conflict of interest.

Open Access This article is distributed under the terms of the Creative Commons Attribution License which permits any use, distribution, and reproduction in any medium, provided the original author(s) and the source are credited.

\section{References}

Bailey TL, Boden M, Buske FA, Frith M, Grant CE, Clementi L, Ren J, Li WW, Noble WS (2009) MEME suite: tools for motif discovery and searching. Nucl Acids Res 37:W202-W208

Bjorklund AK, Ekman D, Light S, Frey-Skott J, Elofsson A (2005) Domain rearrangements in protein evolution. $\mathrm{J}$ Mol Biol 353:911-923

Buljan M, Bateman A (2009) The evolution of protein domain families. Biochem Soc Trans 37:751-755

Casson SA, Chilley PM, Topping JF, Evans IM, Souter MA, Lindsey K (2002) The POLARIS gene of Arabidopsis encodes a predicted peptide required for correct root growth and leaf vascular patterning. Plant Cell 14:1705-1721

Chae YK, Tonneli M, Markley JL (2012) Recombinant production, isotope labeling and purification of ENOD40B: a plant peptide hormone. Protein Pept Lett 19:808-811

Charon C, Sousa C, Crespi M, Kondorosi A (1999) Alteration of enod40 expression modifies Medicago truncatula root nodule development induced by Sinorhizobium meliloti. Plant Cell 11:1953-1966

Chilley PM, Casson SA, Tarkowski P, Hawkins N, Wang KL, Hussey PJ, Beale M, Ecker JR, Sandberg GK, Lindsey K (2006) The POLARIS peptide of Arabidopsis regulates auxin transport and root growth via effects on ethylene signaling. Plant Cell 18:3058-3072

Clough SJ, Bent AF (1998) Floral dip: a simplified method for Agrobacterium-mediated transformation of Arabidopsis thaliana. Plant J 16:735-743

Combier JP, Helge Kuster, Journet EP, Hohnjec N, Gamas P, Niebel A (2008) Evidence for the involvement in nodulation of the two small putative regulatory peptide-encoding genes MtRALF1 and MtDVL1. Mol. Plant Microbe Interact 21:1118-1127

Crespi MD, Jurkevitch E, Poiret M, d'Aubenton-Carafa Y, Petrovics G, Kondorosi E, Kondorosi A (1994) Enod40, a gene expressed during nodule organogenesis, codes for a non-translatable RNA involved in plant growth. EMBO J 13:5099-5112

Farrokhi N, Whitelegge JP, Brusslan JA (2008) Plant peptides and peptidomics. Plant Biotech J 6:105-134

Felsenstein J (1985) Confidence limits on phylogenies: an approach using the bootstrap. Evolution 39:783-791

Fletcher JC, Brand U, Running MP, Simon R, Meyerowitz EM (1999) Signaling of cell fate decisions by CLAVATA3 in Arabidopsis shoot meristems. Science 283:1911-1914 
Floyd SK, Bowman JL (2007) The ancestral developmental tool kit of land plants. Int J Plant Sci 168:1-35

Gamborg OL, Murashige T, Thorpe TA, Vasil IK (1976) Plant tissue culture media. In Vitro 12:473-478

Golz JF, Hudson A (2002) Signaling in plant lateral organ development. Plant Cell 14(Suppl):S277-S288

Huffaker A, Pearce G, Ryan CA (2006) An endogenous peptide signal in Arabidopsis activates components of the innate immune response. Proc Natl Acad Sci USA 103:10098-10103

Ikeuchi M, Tamaguchi T, Kazama T, Ito T, Horiguchi G, Tsukaya H (2010) ROTUNDIFOLIA4 regulates cell proliferation along the body axis in Arabidopsis shoot. Plant Cell Physiol 52:59-69

International Rice Genome Sequencing Project (2005) The map-based sequence of the rice genome. Nature 436:793-800

Jones DT, Taylor WR, Thornton JM (1992) The rapid generation of mutation data matrices from protein sequences. Comput Appl Biosci 8:275-282

Kachroo A, Schopfer CR, Nasrallah ME, Nasrallah JB (2001) Allelespecific receptor ligand interactions in Brassica self-incompatibility. Science 293:1824-1826

Kastin AJ (2013) Handbook of biologically active peptides, 2nd (ed.) Elsevier, San Diego, CA, 1942 pages with 64 plates

Katsir L, Davies KA, Bergmann DC, Laux T (2011) Peptide signaling in plant development. Curr Biol 21:356-364

Kouchi H, Hata S (1993) Isolation and characterization of novel nodulin cDNAs representing genes expressed at early stages of soybean nodule development. Mol Gen Genet 238:106-119

Kumagai H, Kinoshita E, Ridge RW, Kouchi H (2006) RNAi knockdown of ENOD40s leads to significant suppression of nodule formation in Lotus japonicus. Plant Cell Physiol 47:1102-1111

Li L, Li C, Howe GA (2001) Genetic analysis of wounding signaling in Tomato: evidence for a dual role of Jasmonic acid in defense and female fertility. Plant Physiol 127:41414-41417

Matsubayashi Y (2014) Posttranslationally modified small-peptide signals in plants. Annu Rev Plant Biol 65:385-413

Matsubayashi Y, Sakagami Y (2006) Peptide hormones in plants. Annu Rev Plant Biol 57:649-674

Matsuzaki Y, Ogawa-Ohnishi M, Mori A, Matsubayashi Y (2010) Secreted peptide signals required for maintenance of root stem cell niche in Arabidopsis. Science 329:1065-1067

Meindl T, Boller T, Felix G (1998) The plant wound hormone systemin binds with the N-terminal part to its receptor but needs the C-terminal part to activate it. Plant Cell 10:1561-1570

Mihara M, Itoh T, Izawa T (2009) SALAD database: a motif-based database of protein annotations for plant comparative genomics. Nucl Acids Res 38:835-842

Narita NN, Moore S, Horiguchi G, Kubo M, Demura T, Fukuda H, Goodrish J, Tsukaya H (2004) Overexpression of a novel small peptide ROTUNDIFOLIA4 decreases cell proliferation and alters leaf shape in Arabidopsis thaliana. Plant J 38:699-713

Narváez-Vásquez J, Ryan CA (2004) The cellular localization of prosystemin: a functional role for phloem parenchyma in systemic wound signaling. Planta 218:360-369

Narváez-Vásquez J, Pearce G, Ryan CA (2005) The plant cell wall matrix harbors a precursor of defense signaling peptides. Proc Natl Acad Sci USA 102:12974-12977

Pearce G, Strydom D, Johnson S, Ryan CA (1991) A polypeptide from tomato leaves induces wound-inducible proteinase inhibitor proteins. Science 253:895-897

Petricka JJ, Winter CM, Benfey PN (2012) Control of Arabidopsis root development. Annu Rev Plant Biol 63:563-590

Podkowinski J, Zmienko A, Florek B, Wojciechowski P, Rybarczyk A, Wrzesinski J, Ciesiolka J, Blazewicz J, Kondorosi A, Crespi M, Legocki A (2009) Translational and structural analysis of the shortest legume ENOD40 gene in Lupinus luteus. Acta Biochim Polon 56:89-102

Röhrig H, Schmidt J, Miklashevichs E, Schell J, John M (2002) Soybean ENOD40 encodes two peptides that bind to sucrose synthase. Proc Natl Acad Sci USA 99:1915-1920

Röhrig H, John M, Schmidt J (2004) Modification of soybean sucrose synthase by S-thiolation with ENOD40 peptide A. Biochem Biophys Res Commun 325:864-870

Sakagami Y (2007) Plant peptide signals. eLS. doi:10.1002/9780470015902.a0020110

Scheer JM, Ryan CA (2002) The systemin receptor SR160 from Lycopersicon peruvianum is a member of the LRR receptor kinase family. Proc Natl Acad Sci USA 99:9585-9590

Schopfer CR, Nasrallah ME, Nasrallah JB (1999) The male determinant of self incompatibility in Brassica. Science 286:1697-1700

Shimodaira H (2002) An approximately unbiased test of phylogenetic tree selection. Syst Biol 51:492-508

Shimodaira H (2004) Approximately unbiased tests of regions using multistep-multiscale bootstrap resampling. Ann Stat 32:2616-2641

Takeda N, Okamoto S, Hayashi M, Murooka Y (2005) Expression of LjENOD40 genes in response to symbiotic and non-symbiotic signals: LjENOD40-1 and LjENOD40-2 are differentially regulated in Lotus japonicus. Plant Cell Physiol 46:1291-1298

Tamura K, Stecher G, Peterson D, Filipski A, Kumar S (2013) Mega 6: molecular evolutionary genetics analysis version 6.0. Mol Biol Evol 30:2725-2729

The Arabidopsis Genome Initiative (2000) Analysis of the genome sequence of the flowering plant Arabidopsis thaliana. Nature 408:796-815

Tsuge T, Tsukaya H, Uchimiya H (1996) Two independent and polarized processes of cell elongation regulate leaf blade expansion in Arabidopsis thaliana (L.) Heynh. Development 122:1589-1600

Tsukaya H (2006) Mechanism of leaf-shape determination. Annu Rev Plant Biol 57:477-496

Valdivia ER, Chevalier D, Sampedro J, Taylor I, Niederhuth CE, Walker JC (2012) DVL genes play a role in the coordination of socket cell recruitment and differentiation. J Exp Bot 63:1405-1412

Wan X, Hontelez J, Lillo A, Guarnerio C, van de Peut D, Fedorova E, Bisseling T, Franssen H (2007) Medicago truncatula ENOD40-1 and ENOD40-2 are both involved in nodule initiation and bacteroid development. J Exp Bot 58:2033-2041

Weiner J 3rd, Beaussart F, Bornberg-Bauer E (2006) Domain deletions and substitutions in the modular protein evolution. FEBS $\mathrm{J}$ 273:2037-2047

Wen J, Lease KA, Walker JC (2004) DVL, a novel class of small polypeptides: overexpression alters Arabidopsis development. Plant J 37:668-677

Yamaguchi Y, Huffaker A (2011) Endogenous peptide elicitors in higher plants. Curr Opin Plant Biol 14:351-357

Yamaguchi Y, Pearce G, Ryan CA (2006) The cell surface leucinerich repeat receptor for AtPep1, an endogenous peptide elicitor in Arabidopsis, is functional in transgenic tobacco cells. Proc Natl Acad Sci USA 103:10104-10109

Yamaguchi T, Ikeuchi M, Tsukaya H (2013) Chapter 11, ROTUNDIFOLIA4. In: Kastin AJ (ed) Handbook of biologically active peptides, 2nd edn. Elsevier, San Diego, pp 53-57

Yang WC, Katinakis P, Hendriks P, Smolders A, de Vries F, Spee J, van Kammen A, Bisseling T, Franssen H (1993) Characterization of GmENOD40, a gene showing novel patterns of cellspecific expression during soybean nodule development. Plant J 3:573-585 